\title{
Trajectories of Treatment Response in Hallucinations
}

Igne Sinkeviciute ${ }^{1,4,5 *}$, Rolf Gjestad ${ }^{1,5}$, Eirik Kjelby ${ }^{1}$, Laimonas Ratkus, Kenneth Hugdahl ${ }^{1,3,4}$, Rune A. Kroken ${ }^{1,2,4}$, ElseMarie Løberg ${ }^{1,6,7}$, Hugo A. Jørgensen ${ }^{2}$, Iris E Sommer ${ }^{3,8}$, Erik Johnsen ${ }^{1,2,4}$

\begin{abstract}
Objective

Hallucinations are highly prevalent in schizophrenia and related disorders. Antipsychotics are generally effective in treating hallucinations, but major inter-individual differences in treatment response exist. Previous studies have identified heterogeneity of over-all antipsychotic response patterns in schizophrenia. The aim of this study was to explore the heterogeneity in the response of hallucinations to antipsychotic drug treatment in a representative sample of patients acutely admitted for psychosis.
\end{abstract}

\section{Methods}

226 adult patients with symptoms of active psychosis were included in a randomized pragmatic trial of second-generation antipsychotics and followed for 27 weeks. Latent-mixture and latent growth curve models were conducted to analyze heterogeneity of treatment response for hallucinations to second-generation antipsychotics.

\section{Results}

We found five different trajectories of treatment response for patients with hallucinations at baseline. These included two groups of "dramatic responders" who had rapid reduction followed by extinction of hallucinations during the first four weeks of treatment, then groups of "gradual responders", "temporal responders" and "non-responders". Most responders, 80\% of those with hallucinations at baseline, were dramatic responders. Patients who showed no response in the early weeks remained non-responders also after longer follow-up.

\section{Conclusions}

The study suggests the existence of differential response patterns of hallucinations to antipsychotic treatment, and that a significant subgroup are dramatic responders. Hallucinations generally respond quickly to antipsychotic treatment. With no improvement in the very first weeks an early change of treatment should be considered.

\section{Keywords}

Hallucinations, antipsychotics, treatment response, trajectories, psychosis, heterogeneity, schizophrenia

\footnotetext{
'NORMENT, Division of Psychiatry, Haukeland University Hospital, PB 1400, 5021 Bergen, Norway

2Department of Clinical Medicine, Section of Psychiatry, Faculty of Medicine and Dentistry, University of Bergen, Norway

${ }^{3}$ Department of Biological and Medical Psychology, University of Bergen, Norway

${ }^{4}$ Centre for Research and Education in Forensic Psychiatry, Haukeland University Hospital, Bergen, Norway

${ }^{5}$ Department of Addiction Medicine, Haukeland University Hospital, 5021 Bergen, Norway;

${ }^{6}$ Department of Clinical Psychology, University of Bergen, Norway

`University Medical Center Groningen, Department of Neuroscience, Groningen, The Netherlands

${ }^{\dagger}$ Author for correspondences: Igne Sinkeviciute, Division of Psychiatry, Haukeland University Hospital, PB 1400, 5021 Bergen, Norway, Tel: +47 454293 53; Fax: +47 559584 02; email: igne.sinkeviciute@helse-bergen.no
} 


\section{Introduction}

Response to antipsychotic drugs is typically reported dichotomously as rates, and considerable variations are found in recent meta-analyses depending on the specific response criteria used $[1,2]$. However, this responder/ non-responder dichotomy does not capture the range of responses that can be observed in clinical care. A different approach has been to investigate the heterogeneity of antipsychotic response patterns, where different response trajectories have indeed been identified across studies [3-8]. Several issues related to sample characteristics and methodological considerations applied. First, the majority of trajectory studies include patients with chronic schizophrenia which are unlikely to be generalizable to first episode and earlyphase patients (8). Secondly, most studies include selected samples, use of experimental designs at odds with usual clinical practice and short study durations which may further limit generalizability [9]. Finally, most if not all previous studies use joint total scores of several symptom clusters or sum scores of single symptoms clusters as outcome measures $[10,11]$. By focusing on sum scores there is a risk to overlook the heterogeneity in treatment response in any particular symptom, as this might be cancelled out by opposing changes in other symptoms. A new approach would be to focus on a single symptom (see [12] for a discussion of single symptom approach in psychiatry research). Although sharing the same reality breach, psychotic disorders are heterogeneous symptomatically as demonstrated in the various diagnostic manuals $[13,14]$.

Hallucinations are prevalent in schizophrenia and related disorders [15]. As much as 80 $\%$ of patients with schizophrenia experience hallucinations where auditory hallucinations are most frequent [15]. Hallucinations may cause substantial suffering and are sometimes associated with suicidality [16-19], violence $[20,21]$ and homicide [22]. Hallucinations are therefore symptoms of both major clinical importance and with distinguishing properties.

We have previously demonstrated that differential effectiveness may exist among second generation antipsychotics for hallucinations using a pragmatic RCT with a 24-months follow-up period [23]. Corresponding findings were also reported in a larger study [24]. An important new research question relates to whether different response trajectories of hallucinations exist independently of the particular antipsychotic drugs used.
The aims of the study were to investigate the heterogeneity of hallucinatory response to antipsychotic drug treatment in a representative sample of patients acutely admitted for psychosis.

\section{Material and Methods \\ - Study design}

The present study uses data from the Bergen Psychosis Project (BPP), a 24-month prospective, randomized, pragmatic antipsychotic drug trial. The design and methods have been described in more detail elsewhere [25]. The study was conducted between 2004 and 2009 at the Division of Psychiatry, at Haukeland University Hospital in Bergen, Norway. The hospital serves a catchment population of about 400,000 . The project was approved by the Regional Committee for Medical Research Ethics $n$ Western Norway (REK Vest) and the Norwegian Social Science Data Services (NSD). The Regional Committee for Medical Research Ethics allowed eligible patients to be included before informed consent was provided, which facilitates a clinically relevant representation in the study. The BPP was publicly funded and did not receive any financial or other support from the pharmaceutical industry. The present study focuses on the first six months of follow-up.

\section{- Sample}

The sample was consecutively recruited from the psychiatric emergency ward. Adult patients were eligible for the study if they were admitted for symptoms of active psychosis as determined by a score of $\geq 4$ on one or more of the items Delusions, Hallucinations, Grandiosity, Suspiciousness/persecution or Unusual thought content in the Positive and Negative Syndrome Scale (PANSS) [26] and were candidates for oral antipsychotic drug therapy. All included patients met the diagnostic criteria of the International Classification of Disease ICD-10 [13] for schizophrenia, schizoaffective disorder, acute and transient psychotic disorder, delusional disorder, drug-induced psychosis, major depressive disorder with psychotic features and bipolar disorder except manic psychosis.

Patients with drug-induced psychosis were only included when the condition did not resolve within a few days and when antipsychotic drug therapy was indicated. The diagnoses were determined by the psychiatrists or specialists in clinical psychology in the hospital. Exclusion criteria were inability to use oral antipsychotics, 
inability to cooperate during the investigation because of manic psychosis or other behavioral or mental reasons related to the state of illness, not understanding Norwegian language, treatment with clozapine at admission or being candidate for electroconvulsive therapy treatment.

A total of 226 adult (> 18 years) patients were at inclusion randomized to one of the following antipsychotics: risperidone, olanzapine, quetiapine or ziprasidone. In accordance with a pragmatic design aiming to mimic everyday clinical decision making, the randomization was open to both the patient and the clinical staff, whereas the assessments were conducted by blinded research personnel. Furthermore, the patients were randomized to a random sequence of the investigational agents rather than a single drug. The patients were offered the first agent in the sequence but could choose the next agent in the sequence if the first drug could not be used because of contraindications or prior negative experience. The same procedure was followed if the next antipsychotic $\operatorname{drug}(\mathrm{s})$ in the sequence could not be used. The treating psychiatrist was free to choose the dosing, combination with other psychotropics and discontinuation and change to another antipsychotic agent in the presence of insufficient efficacy and/ or side effects. In the rare case that a patient already used an antipsychotic agent in therapeutic dosage at admission, no wash-out was performed before starting the study drug. If a patient was randomized to the same agent already under use, the agent would simply be continued and only dose adjustment made if indicated.

\section{- Measurements}

The patients were assessed at baseline (T1), at discharge or latest at 9 weeks (mean 4.1 weeks) if not discharged earlier (T2) and at follow-up visits after 3 (T3) and 6 months (T4). The main reason for drop-out between $\mathrm{T} 1$ and $\mathrm{T} 2$ was discharge from the hospital before one week had passed since baseline, which was defined as the minimum interval before T2 assessments could be undertaken. There were no baseline differences in any clinical or demographic variables between those with baseline assessments only and those with follow up assessments, except for higher PANSS negative subscale score in those with baseline assessments only (independent samples t-test: $\mathrm{p}=0.02 ;$ mean difference $2.3 ; 95 \%$ confidence interval of the difference 0.4-4.2). The severity of hallucinations was evaluated by item P3 (Hallucinatory behavior) of the PANSS positive subscale, where a score of 3 or more indicate the presence of hallucinations. The Intraclass correlation coefficient (ICC) for the PANSS was calculated based on inter-rater assessments between EJ, HAJ and RAK, and showed high inter-rater reliability (0.92). Calgary Depression Scale for Schizophrenia (CDSS) was used to measure severity of depression [27]. CDUS/ CAUS - the Clinical Drug and Alcohol Use Scales - were conducted for overview of the use of alcohol and illicit drugs [28]. The Clinical Global Impression-Severity of Illness scale (CGI-I) [29] and Global Assessment of Functioning-Split Version, Functions scale (GAF-F) were used to assess the general functioning level [30]. A brief neuropsychological screening instrument; the Repeatable Battery for the Assessment of Neuropsychological Status (RBANS), was administered at baseline [31,32]. Serum level measurements of antipsychotic medication were performed at all follow-up visits.

\section{- Statistical data analysis}

SPSS 22 was used for descriptive statistics, frequency, cross tabulation with chi-square and group difference t-test [33]. Level and change at mean and individual level was analyzed with latent growth curve modeling (LGC) [34]. The outcome variable (PANSS item P3) was ordinal; however, with seven categories and therefore analyzed as a continuous variable. The Maximum Likelihood Estimator with robust standard errors (MLR) was used in order to give unbiased estimates when non-normality in data was experienced [35]. The Full Information Maximization Likelihood method uses all available data under the "Missing at Random" assumption [35,36], minimizes the negative effect of missing data, improves statistical power and generalizability of the results compared to models based on the ordinary listwise deletion method [37].

An LGC-model from $\mathrm{T} 1$ to $\mathrm{T} 4$ revealed estimation problems caused by the linearity restriction. A quadratic factor was added in order to describe a non-linear change over time. However, this model did not fit the data (CFI=0.58, TLI=0.37, RMSEA=0.18) and showed estimations problems as well. These results together with a visual inspection of the observed data showed further needs for improvements. In the final model one linear difference factor that described level and change from T1 to T2 after approximately four weeks (S: $0-4.1$ weeks) and another latent factor 
capturing the change from T2 to T4 (S2: 4.127.0 weeks) was estimated.

Then, Latent Class Growth Analysis (LCGA) was conducted to describe the heterogeneity in the data expressed as the maximal number of possible trajectories [38]. The evaluation of the number of classes was based on the entropy index and model fit indices Akaike Information Criterion (AIC), the Bayesian Information Criterion (BIC) and sample-adjusted BIC, with lower values indicating better model fit $[35,39]$. Statistically significant improvement of adding classes was tested by the Vuong-Lo-Mendell-Rubin Likelihood Ratio Test for $\mathrm{k}-1$ versus $\mathrm{k}$ classes (LRT) [39]. The estimated class size and relative frequencies were used in this evaluation. A sample size of 25 classified subjects and $5 \%$ of the sample has been proposed as threshold values [40]. Mplus 7.4 was used for LGC analysis and LCGA [36].

\section{Results}

A total of 226 patients were included, of which $74(32.7 \%)$ were females. The mean age at baseline was 34.1 (SD 13.5) and 44.2\% had not used antipsychotic drugs before. The distribution of the study-drugs at baseline was $23.6 \%$ risperidone, $29.8 \%$ olanzapine, $21.8 \%$ quetiapine and $24.9 \%$ ziprasidone. At the last follow up the distribution of medications was similar.

The descriptive statistics and sample size for the outcome variable at baseline (T1), at first followup (mean 4.1 weeks after baseline) (T2) and at follow-up visits after 3 (T3) and 6 months (T4) are presented in Table 1 . The use of antipsychotic drugs and concomitant psychotropic medications are displayed in the supplementary material, Supplementary Table 1.

- Level and change in hallucinations over time in the total group

Latent growth curve (LGC) analyses were used to investigate the level and change in the hallucination score for the total sample collectively. After exploring different models, one linear change factor that described level and change from T1 to T2 (S1: 0-4 weeks) and another factor capturing the change from $\mathrm{T} 2$ to T4 (S2: 4-27 weeks), were estimated. The mean baseline PANSS P3 score was estimated to be $3.51(\mathrm{p}<0.001)$ with individual variance $=2.65$ (SD 1.63), the mean change from $\mathrm{T} 1$ to $\mathrm{T} 2$ was -0.38 per week $(\mathrm{p}<0.001)$ and the latent change from T2 to T4 was -0.01 per week $(p=0.06)$. The individual variance in change for the two slope factors were $\mathrm{S} 1: \mathrm{SD}=0.38(\mathrm{p}<0.01)$ and $\mathrm{S} 2: \mathrm{SD}=0.03(\mathrm{p}=0.51)$. Stronger reductions in the scores from T1 to T2 were seen in patients with higher baseline scores than in patients with lower baseline scores $(r=-0.71, p<0.001)$. This model fitted data well $\left(x^{2}=2.63, d f=2, p=0.27\right.$, Comparative fit index $(\mathrm{CFI})=0.99$, TuckerLewis Index $(T L I)=0.97$, Root mean square error of approximation (RMSEA) $=0.037$, RMSEA $\mathrm{CI}=0.000-0.143$, RMSEA close fit=0.45).

\section{- Description of different trajectories}

Latent class analyses (LCGA) revealed up to seven trajectory classes (Table 2). The LRTand entropy results indicated three and seven classes as the best models. The smallest class in the three class model was larger in size than in the seven class model. However, the seven class model more precisely captured the individual variability in level and changes and was therefore chosen for presentations. The model is presented in Figure 1 and show trajectories with different baseline levels and changes of hallucinations: one high hallucination-level class that maintain the symptom level during the follow up (C1: $3 \%$ of the sample); one class with initially high hallucination scores with a temporary improvement and then worsening of the symptom level (C2: 4\%); two large classes with early improvements (C3: 30\%) and (C7: 24\%); one low hallucination-level class with worsening of the symptom at 4 weeks and then improvement (C4: 3\%); a low hallucination-level class that maintain the same level during the follow-up (C5: 29\%) and finally a high hallucination-level class with slow, but progressive reduction of the hallucinations score (C6: 6\% of the sample).

\section{- Hallucinating subgroup}

Three groups of patients with hallucinations at baseline had marked reductions of hallucination scores, of which two groups $(80 \%$ of those with hallucinations at baseline) might be categorized as "dramatic responders". This group had a rapid reduction followed by extinction of hallucinations during the first four weeks of treatment. One group - $(10 \%$ of those with hallucinations) showed a more gradual but ultimately strong response and might be categorized as "gradual responders" One group (7\% collectively of those with hallucinations) had temporary reduction of hallucinations and might be categorized as "temporal responders". Non-responders $(4 \%$ collectively of those with hallucinations) were patients who had no reduction of the hallucination score. 


\begin{tabular}{|c|c|c|c|c|}
\hline & $\mathrm{N}$ & Mean & SD & Skewness \\
\hline T1 & 226 & 3.51 & 1.63 & -0.43 \\
\hline $\mathrm{T} 2$ & 109 & 2.03 & 1.42 & 1.05 \\
\hline T3 & 52 & 1.73 & 1.29 & 1.51 \\
\hline $\mathrm{T} 4$ & 39 & 1.85 & 1.48 & 1.41 \\
\hline
\end{tabular}

\begin{tabular}{|c|c|c|c|c|c|c|}
\hline Classes & AIC & BIC & SABIC & $\begin{array}{l}\text { LRT } \\
p \text {-value }{ }^{a}\end{array}$ & Entropy & $\begin{array}{l}\text { Least } \\
\text { class } \\
\text { predicted n (\%) }\end{array}$ \\
\hline 1 & 1575 & 1599 & 1576 & - & - & \\
\hline 2 & 1418 & 1455 & 1420 & 0.014 & 0.58 & 34 (15\%) \\
\hline 3 & 1320 & 1372 & 1324 & 0.000 & 0.74 & $33(15 \%)$ \\
\hline 4 & 1286 & 1351 & 1290 & 0.173 & 0.70 & $4(2 \%)$ \\
\hline 5 & 1259 & 1338 & 1268 & 0.274 & 0.71 & $3(1 \%)$ \\
\hline $6^{b}$ & 1226 & 1318 & 1233 & 0.012 & 0.73 & $6(3 \%)$ \\
\hline $7^{c}$ & 1213 & 1319 & 1221 & 0.031 & 0.75 & $6(3 \%)$ \\
\hline $8^{d}$ & 1148 & 1268 & 1157 & 0.270 & 0.71 & $4(2 \%)$ \\
\hline
\end{tabular}

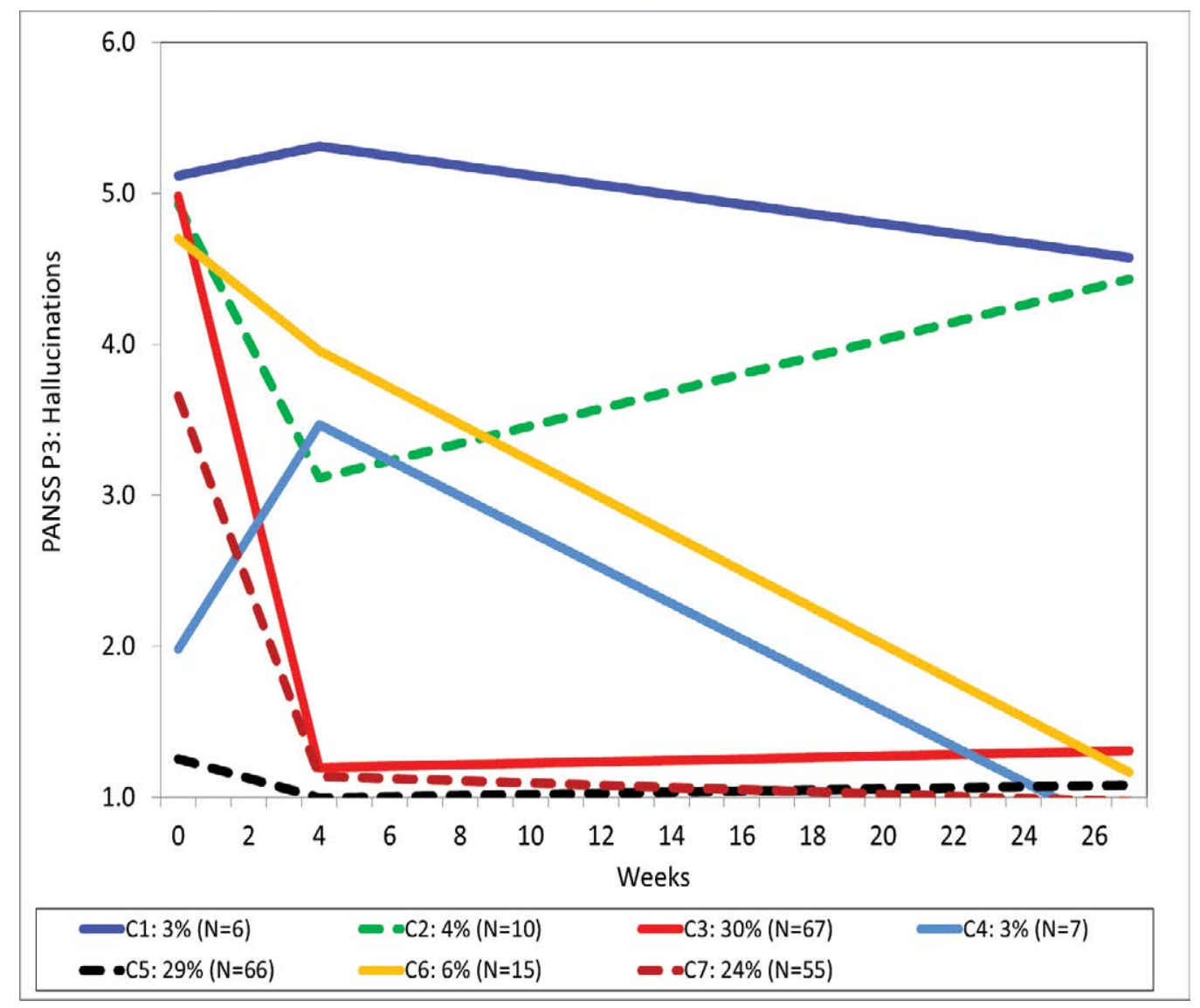

Figure 1: Results from Latent Class Growth Analysis (LCGA) showing level and changes over time in hallucinatory behavior for seven classes/ trajectories (C1-C7). Frequencies for the most likely latent class membership are given. 


\section{Discussion}

The main objective of the study was to disentangle different response trajectories of hallucinations as a prevalent and clinically relevant symptom. Seven different response patterns were identified, five of those included patients with hallucinations at baseline. More than three quarters of patients with clinically significant hallucinations at baseline could be defined as dramatic responders, with extinction of hallucinations during the first four weeks of treatment. This shows that hallucinations tend to respond rapidly and well to antipsychotic medication in the vast of majority of psychotic patients troubled by this symptom. In the shared decision process, this information is relevant to patients and caregivers.

Most studies that have investigated heterogeneity in treatment response, have focused on total psychopathology or one of the symptom sub-dimensions which complicates direct comparisons of our results to other studies. However, studies typically report four to five distinct response patterns. Marques and colleagues [5] found four response trajectories for positive symptoms during six weeks of follow-up. These are categorized as "dramatic responders" (patients that start off with a high symptom level and show dramatic decrease of symptoms), "responders" (patients that start off with average symptom scores and show robust response), "partial responders" (patients that show modest response) and finally "non-responders". PelayoTeran and colleagues [11] found five trajectories for positive symptoms response during six weeks of follow-up. Comparable terminology is used among the studies for the different trajectories, including also a "slow partial responders" group in the latter study. Levine and colleagues [6] report response in total psychopathology during eight weeks of follow-up and fond groups of "poor responders", "rapid treatment responders" and three groups of "moderate responders".

The proportions with non- or poor response are quite similar across studies, with $10 \%$ reported by Marques et al [5], 8,3\% reported by PelayoTeran et al [11], 8\% reported by Levine et al [6]. In our study non responders reached $7 \%$ after 6 month in the total sample and $11 \%$ in the hallucinating subgroup, however part of those patients were temporal responders, with rapid and significant response in the first 4 weeks, followed by gradual worsening of hallucinations.
The group that could be characterized as "dramatic responders" was significantly larger in our study compared to the other studies $[5,11]$. Reasons for these differences may include a faster and more pronounced response of hallucinations compared to other symptoms of psychosis, such as for example delusions. Several other studies reports significantly faster response of hallucinations to antipsychotic treatment than that for delusions $[41,42]$. In this context, the delayed response of delusions to antipsychotic drug treatment would also influence the sum scores of positive symptoms in which delusions are embedded, causing the change of sum scores to appear at a slower rate.

Our results may be related to the pragmatic design of our study with a more clinically relevant sample compared to those in most RCTs. Indeed findings from the EUFEST study, a large pragmatic trial of antipsychotic effectiveness, found a substantially larger PANSSscore reduction compared to that reported in traditional randomized trials of antipsychotic efficacy [43], which among others include less diverse study populations because of numerous in- and exclusion criteria [44].

Results may also be related to our sample of patients in the acute phase of psychosis. Faster reduction of symptom would be expected in this patient group compared to more stable patients.

Our study revealed a small but clinically important group of patients $(3 \%$ of the total sample) who had sub-threshold scores for hallucinations at baseline, but then had an apparent worsening of symptoms, followed by a gradual decline and extinguishing of hallucinations. We can only speculate about the reasons for this particular response trajectory, but might include actual worsening of hallucinations during the acute phase or under-reporting of symptoms at baseline.

Results from our study might support the notion of different underlying mechanisms for the hallucinations. Findings in our study, where most of the patient are dramatic responders, might also support underlying pathophysiological dopaminergic mechanism, where high levels of dopamine in the limbic system play a major role, in most of the cases with hallucinations. However, non-responders might have a different underlying mechanism for the hallucinations [45] and other treatment options than D2receptor blocking antipsychotics should be considered. Unfortunately, as of yet, not many other treatment options in the clinical care are 
available as all of the antipsychotics primarily blocks D2 receptors (for discussion about personalized treatment of hallucinations see I. Sommer et al [45]).

Another important aspect of different response trajectories is their relation to clinical and non-clinical characteristics. Factors such as diagnosis, other symptoms, previous medication or different metabolism of the drugs, as well as gender, genetic variation, nutrition, specific diets or ethnicity might play a role in treatment response and thereby potentially serve as predictors of different response patterns [4648]. Investigating such predictors was beyond the scope of this paper, however, but should be a focus in the future studies.

\section{Limitations}

The main advantages of the study are the consecutive recruitment of a clinically representative acute-phase sample and the focus on a single symptom of high prevalence and clinical significance, both of which are compliant with the recently suggested strategy for new drug developments in psychosis [49]. Some limitations should nevertheless be mentioned: High dropout rates are an important and wellknown issue in antipsychotic drug trials [50]. The current study had a considerable attrition rate, but was comparable to other RCTs conducted on schizophrenia spectrum disorders [51]. Also, attrition did not seem to be associated with any baseline characteristics except a slightly higher PANSS negative subscale score in those with baseline assessments only. Choosing seven classes in LCGA model without reaching a proposed sample size of 25 classified subjects and five percent of the sample represent a limitation. We attempted to identify the highest number of highly homogeneous groups to represent the heterogeneity seen in every day clinical life, but because some of the classes were small, further statistical analyses of inter-class differences were not performed. These results need to be replicated in samples of larger size before any final conclusions can be drawn. Finally, hallucinations were investigated collectively as we did not have information beyond the hallucinations item of the PANSS scale. Theoretically, different modalities of hallucinations either might have individual response patterns, and future studies should include subtyping of hallucination modalities such as auditory, visual, tactile, and others.

\section{Conclusions}

This study contributes with new evidence concerning hallucinations as a separate target for antipsychotic drug treatment and a basis for stratified approaches. Hallucinations generally respond quickly to antipsychotic drug treatment and in the absence of response during the very first weeks of treatment, an early change of treatment should be considered. Future studies should aim at identifying predictors of the different treatment trajectories to better individualize treatment.

\section{Conflicts of Interest}

Iris Sommer is consultant to Gabather and has received research support from Janssen and $\mathrm{Su}$ novion.

\section{Acknowledgments}

Contribution of co-author $\mathrm{KH}$ was funded by ERC AdG \# 693124

\section{Author contributions}

All authors made substantive intellectual contributions to the study. IS planned the study, wrote the first draft of the manuscript and participated in the statistical analyses; RG undertook the statistical analyses, contributed in the interpretations of the data and helped draft the manuscript; EK contributed in the interpretations of the data and helped draft the manuscript; LR contributed in the interpretations of the data and helped draft the manuscript; $\mathrm{KH}$ contributed in the interpretations of the data and helped draft the manuscript; RAK collected data, contributed in analyses and interpretations of the data and helped draft the manuscript; EML contributed in analyses and interpretations of the data and helped draft the manuscript; HAJ designed the study, assisted in data collection, contributed in analyses and interpretations of the data and contributed to the drafting of the manuscript; EJ was the co-designer of the study, collected data, undertook the statistical analyses and helped draft the manuscript. All authors have read and given final approval of the latest version of the manuscript. 


\section{References}

1. Zhu Y, Li C, Huhn M, et al. How well do patients with a first episode of schizophrenia respond to antipsychotics: A systematic review and meta-analysis. Eur. Neuropsychopharmacology: J. Eur. Coll. Neuropsychopharmacology 27(9), 835-844 (2017).

2. Leucht $S$, Leucht $C$, Huhn M, et al. Sixty Years of Placebo-Controlled Antipsychotic Drug Trials in Acute Schizophrenia: Systematic Review, Bayesian Meta-Analysis, and Meta-Regression of Efficacy Predictors. Am. J. Psychiatry 174(10), 927-942 (2017).

3. Stauffer V, Case M, Kollack-Walker S, et al. Trajectories of response to treatment with atypical antipsychotic medication in patients with schizophrenia pooled from 6 double-blind, randomized clinical trials. Schizophr. Res 130(1-3), 11-19 (2011).

4. Case M, Stauffer VL, Ascher-Svanum H, et al. The heterogeneity of antipsychotic response in the treatment of schizophrenia. Psychological medicine 41(6), 1291-1300 (2011).

5. Marques TR, Arenovich T, Agid O, et al. The different trajectories of antipsychotic response: antipsychotics versus placebo. Psychol. Med 41(7), 1481-1488 (2011).

6. Levine SZ, Leucht S. Elaboration on the early-onset hypothesis of antipsychotic drug action: treatment response trajectories. Biol. Psychiatry 68(1), 86-92 (2010).

7. Levine SZ, Leucht S. Early symptom response to antipsychotic medication as a marker of subsequent symptom change: an eighteen-month follow-up study of recent episode schizophrenia. Schizophr. Res 141(2-3), 168-172 (2012).

8. Levine SZ, Rabinowitz J, Faries D, et al. Treatment response trajectories and antipsychotic medications: examination of up to 18 months of treatment in the CATIE chronic schizophrenia trial. Schizophr. Res 137(1-3), 141-146 (2012).

9. March JS, Silva SG, Compton S, et al. The case for practical clinical trials in psychiatry. Am. J. Psychiatry 162(5), 836-846 (2005).

10. Schennach R, Meyer S, Seemuller F, et al. Response trajectories in "real-world" naturalistically treated schizophrenia patients. Schizophr. Res 139(1-3), 218-224 (2012).

11. Pelayo-Teran JM, Diaz FJ, Perez-Iglesias R, et al. Trajectories of symptom dimensions in short-term response to antipsychotic treatment in patients with a first episode of non-affective psychosis. Psychol. Med 44(1), 37-50 (2014).

12. Hugdahl K. Auditory hallucinations: A review of the ERC "VOICE" project. World. J. Psychiatry 5(2), 193-209 (2015).

13. World Health Organisation. International Statistical Classification of Diseases and
Related Health Problems, 10th Revision, Malta (2007).

14. American Psychiatric Association. Diagnostic and Statistical Manual of Mental Disorders: 4th Edtn. American Psychiatric Publishing, Virginia, USA (2000).

15. McCarthy-Jones S, Smailes D, Corvin A, et al. Occurrence and co-occurrence of hallucinations by modality in schizophrenia-spectrum disorders. Psychiatry. Res 252(1), 154-160 (2017).

16. Song JY, Yu HY, Kim SH, et al. Assessment of Risk Factors Related to Suicide Attempts in Patients With Bipolar Disorder. J. Nerv. Ment. Dis 200(11), 978-984 (2012).

17. Madsen T, Nordentoft M. Suicidal changes in patients with first episode psychosis: clinical predictors of increasing suicidal tendency in the early treatment phase. Early. Interv. Psychiatry 6(3), 292-299 (2012).

18. Hor K, Taylor M. Suicide and schizophrenia: a systematic review of rates and risk factors. J. Psychopharmacol 24(S4), 81-90 (2010).

19. Kjelby E, Sinkeviciute I, Gjestad R, et al. Suicidality in schizophrenia spectrum disorders: the relationship to hallucinations and persecutory delusions. Eur. Psychiatry 30(7), 830-836 (2015).

20. Yee NY, Large MM, Kemp RI, et al. Severe non-lethal violence during psychotic illness. Aus. New Zealand. J. Psychiatry 45(6), 466472 (2011).

21. McNiel DE, Eisner JP, Binder RL. The relationship between command hallucinations and violence. Psychiatric. Ser 51(10), 12881292 (2000).

22. Nielssen OB, Westmore BD, Large MM, et al. Homicide during psychotic illness in New South Wales between 1993 and 2002. Med. J. Aus 186(6), 301-304 (2007).

23. Johnsen E, Sinkeviciute I, Loberg EM, et al. Hallucinations in acutely admitted patients with psychosis, and effectiveness of risperidone, olanzapine, quetiapine, and ziprasidone: a pragmatic, randomized study. $B M C$ Psychiatry 13(1), 241 (2013).

24. Sommer IE, Slotema CW, Daskalakis ZJ, et al. The Treatment of Hallucinations in Schizophrenia Spectrum Disorders. Schizophr. Bull 38(4), 704-714 (2012).

25. Johnsen E, Kroken RA, Wentzel-Larsen T, et al. Effectiveness of second-generation antipsychotics: a naturalistic, randomized comparison of olanzapine, quetiapine, risperidone, and ziprasidone. BMC. Psychiatry 10(1), 26 (2010).

26. Kay SR, Fiszbein A, Opler LA. The positive and negative syndrome scale (PANSS) for schizophrenia. Schizophr. Bull 13(2), 261276 (1987).

27. Addington D, Addington J, Schissel B. A depression rating scale for schizophrenics. Schizophr. Res 3(4), 247-251 (1990).

28. Drake RE, Rosenberg SD, Mueser KT. Assessing substance use disorder in persons with severe mental illness. New. Direct. Mental. Health. Ser 70(2), 3-17 (1996).

29. Guy W. Assessment Manual for Psychopharmacology - Revisited. Rockville: US Department of Health and Human Services (1976).

30. Karterud SPG, Loevdahl H, Friis S. Global Assessment of Functioning - Split Version (S-GAF): Background and Scoring Manual. Ullevaal University Hospital, Department of Psychiatry, Oslo, Norway (1998).

31. Gold JM, Queern C, lannone VN, et al. Repeatable battery for the assessment of neuropsychological status as a screening test in schizophrenia I: sensitivity, reliability, and validity. Am. J. Psychiatry 156(12), 19441950 (1999).

32. Randolph C. RBANS Repeatable Battery for the Assessment of Neuropsychological Status: Manual. The Psychological Corporation, San Antonio, Texas (1998).

33. IBM Corp. IBM SPSS Statistics for Windows: Ver 22.0. IBM Corp Armonk, NY (2013).

34. Newsom JT. Longitudinal structural equation modeling. Taylor \& Francis Group, New York (2015).

35. Kline RB. Principles and practice of structural equation modeling: 3rd Edtn. The Guilford Press, New York (2010).

36. Muthén LK, Muthén BO. Mplus 7.3. 3463 Stoner Avenue, Los Angeles, California (2014).

37. Bollen KA, Curran PJ. Latent curve models: A structural equation perspective: Hoboken NJ, 12th Edtn. Wiley-Interscience, USA (2006).

38. Wang J, Wang X. Structural Equation Modeling: Applications Using Mplus West Sussex. A John Wiley \& Sons Publication, UK (2012).

39. Muthén LK, Muthén BO. Mplus. Statistical analysis with latent varibles: User's Guide: Version 7: 7th Edtn. 3463 Stoner Avenue, CA 90066, Los Angeles, 2012.

40. Wickrama KAS, Lee TK, O'Neal CW, et al. Higher-Order Growth Curves and Mixture Modeling with Mplus. Routledge, New York (2016).

41. Schneider SD, Jelinek L, Lincoln TM, et al. What happened to the voices? A finegrained analysis of how hallucinations and delusions change under psychiatric treatment. Psychiatry. Res 188(1), 13-17 (2011).

42. Gunduz-Bruce $H$, McMeniman $M$, Robinson DG, et al. Duration of untreated psychosis and time to treatment response for delu- 


\section{Trajectories of Treatment Response in Hallucinations Research}

sions and hallucinations. Am. J. Psychiatry 162(10), 1966-1969 (2005).

43. Kahn RS, Fleischhacker WW, Boter $\mathrm{H}$, et al. Effectiveness of antipsychotic drugs in first-episode schizophrenia and schizophreniform disorder: an open randomised clinical trial. Lancet 371(9618), 1085-1097 (2008).

44. Tunis SR, Stryer DB, Clancy CM. Practical clinical trials: increasing the value of clinical research for decision making in clinical and health policy. Jama 290(12), 1624-1632 (2003).

45. Sommer IE, Kleijer H, Hugdahl K. Toward personalized treatment of hallucinations. Curr. Opin. Psychiatry 31(3), 237-245 (2018).

46. Emsley RA, Roberts MC, Rataemane S, et al. Ethnicity and treatment response in schizophrenia: a comparison of 3 ethnic groups. J. Clin. Psychiatry 63(1), 9-14 (2002).

47. Chaudhry I, Neelam K, Duddu V, et al. Ethnicity and psychopharmacology. J. Psychopharmacology 22(6), 673-680 (2008).

48. Pouget JG, Shams TA, Tiwari AK, et al. Pharmacogenetics and outcome with antipsychotic drugs. Dialogues. Clin. Neurosci 16(4), 555-566 (2014).
49. Keshavan MS, Lawler AN, Nasrallah HA, et al. New drug developments in psychosis: Challenges, opportunities and strategies. Prog. Neurobiol 152(1), 3-20 (2016).

50. Leucht S, Heres S, Hamann J, et al. Methodological issues in current antipsychotic drug trials. Schizophr. Bull 34(2), 275-285 (2008).

51. Rabinowitz J, Levine SZ, Barkai O, et al. Dropout rates in randomized clinical trials of antipsychotics: a meta-analysis comparing first- and second-generation drugs and an examination of the role of trial design features. Schizophr. Bull 35(4), 775-788 (2009). 